\title{
Gestión del riesgo operacional en el proceso de transporte de producto terminado en el sector
}

\author{
azucarero. \\ Operational risk management in the transport of finished product process in the \\ sugar sector.
}

\author{
M. A. Coronado iD, M. Colorado iD, J. C. Osorio-Gómez iD
}

\begin{abstract}
Risk management in supply chains has become very important, both from academic perspective and form practical applications. The department of Valle del Cauca in Colombia is recognized for its agro-industrial vocation, especially for the sugar production in its sugar mills. Considering the importance of the sugar sector in Valle del Cauca 's economy and the current trend on risk management, a proposal is presented for the identification and priorization of operational risks in the transportation of sugar.

The methodology of risk management applied in this document takes as a reference the organizations involved in the sugar transport process, in order to identify and prioritize the operational risks to propose actions to mitigate or prevent the negative effects that may affect the process The development of the methodology uses a checklist to identify the risks, and the probability and impact matrix, as well as the fuzzy quality function (FQFD) to prioritize them.
\end{abstract}

Index Terms - fuzzy QFD, Risk management, Sugar sector, Transportation of finished product.

Resumen - La gestión del riesgo en las cadenas de suministro ha venido tomando gran importancia, tanto desde la perspectiva académica como de las aplicaciones prácticas. El departamento del Valle del Cauca en Colombia es reconocido por su vocación agroindustrial, destacándose la producción de azúcar en sus ingenios azucareros. Considerando entonces, la importancia del sector azucarero en la economía Vallecaucana y la tendencia actual sobre la gestión de riesgos, se presenta una propuesta para la identificación y priorización de los riesgos operacionales en el transporte del azúcar. La metodología aplicada toma como referencia organizaciones que intervienen en el proceso de transporte de azúcar, con la finalidad de identificar y priorizar

Este manuscrito fue enviado el 30 de agosto de 2019.

${ }^{1}$ M. A. Coronado, Ingeniera Industrial egresada de la Universidad del Valle, Santiago de Cali, Colombia

${ }^{2}$ M. Colorado, Ingeniera Industrial egresada de la Universidad del Valle, Santiago de Cali, Colombia

${ }^{3}$ J. C. Osorio-Gómez, Ingeniero Industrial, Doctor en Ingeniería Industrial de la Universidad del Valle, Santiago de Cali, Colombia, y profesor titular de la Escuela de Ingeniería Industrial los riesgos operacionales que permitan proponer acciones para mitigar o prevenir los impactos negativos que pueden afectar el proceso. El desarrollo de la metodología utiliza una lista de chequeo para la identificación de los riesgos, y la matriz de probabilidad e impacto, además del despliegue de la función de calidad difuso (fuzzy quality function deployment, FQFD) para la priorización de los mismos.

Palabras claves- Gestión del riesgo, QFD difuso, Sector azucarero, Transporte de producto terminado.

\section{INTRODUCCIÓN}

$\mathrm{L}$ AS empresas han reaccionado ante las aparentes oportunidades y amenazas de la globalización a través de varias prácticas de producción global que han aumentado la complejidad de la cadena de suministro y a su vez diversas formas de riesgo [1]. Debido a esto, es indispensable que las organizaciones desarrollen estrategias apropiadas que les permitan entender las causas y anticiparse a esos riesgos. Para ello, lo principal es comprender el funcionamiento de la cadena de suministro, donde el transporte de producto terminado es el proceso que establece el puente entre la empresa y los clientes, este cual puede ser directo o a través de la tercerización, lo que genera vulnerabilidad.

Por su parte, el sector azucarero es un pilar de la economía del departamento del Valle del Cauca con alto impacto social, alcanzando a generar 188.000 empleos y 1.200 .000 personas beneficiadas [2], es así como al garantizar la sostenibilidad del sector se impacta igualmente en el bienestar de la comunidad. Las empresas productoras del sector presentan gestión del riesgo para los procesos productivos, pero no abarca el eslabón del transporte de producto terminado, en el que intervienen diferentes actores y puede ser interrumpido por factores internos o externos.

Es por esta razón que se considera importante la identificación y priorización de los riesgos del proceso mencionado anteriormente; para ello, se tomaron como referencia diferentes organizaciones vinculadas a la cadena de 
este sector entre las que se encuentran productoras $\mathrm{y}$ transportadoras, quienes a partir de su experiencia aportaron a la identificación de los riesgos en el proceso, los cuales se priorizaron con la metodología QFD difuso. A continuación, se presenta la metodología propuesta, en el apartado 3 la aplicación y los resultados de dicha aplicación y finalmente en el apartado 4 las conclusiones.

\section{MetodologíA}

La gestión del riesgo es una pieza clave para la productividad en la generación de valor de las organizaciones y puede ser adoptada como una estrategia competitiva, ya que genera un control basado en planes de contingencia que permite establecer la capacidad para reaccionar a eventos que ocurren bajo el criterio de incertidumbre, el cual incrementa a causa de la variabilidad que surge de la globalización y que afecta la sostenibilidad de la cadena.

El riesgo operacional se define como la posibilidad de que suceda algo que tendrá impacto en el cumplimiento de los objetivos, el impacto puede ser positivo o negativo y se valora en función de las consecuencias como también de las probabilidades de ocurrencia [3]. Para esta metodología se consideran aquellos que tienen un impacto negativo en la organización.

Es importante que la gestión del riesgo se lleve a cabo de una forma estructurada, para ello varios autores concuerdan en proponen las etapas de identificación, evaluación de riesgos, acciones orientadas a la mitigación y el monitoreo de los riesgos y de las acciones propuestas, estas etapas se muestran en la ilustración 1.

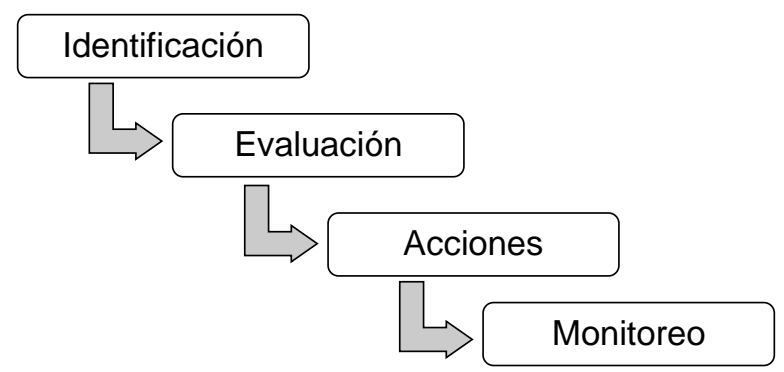

Fig. 1. Fases para la gestión del riesgo.

\section{A. Identificación de los riesgos}

Es punto inicial para la gestión del riesgo operacional y para implementar cualquier tipo de estrategia de mitigación, en la identificación se pretende descubrir, definir y documentar los riesgos claves y su vinculación con los objetivos de la organización [4]. La recolección de datos, las entrevistas y los cuestionarios con herramientas útiles para la identificación de los riesgos.

En esta etapa se parte de la revisión de literatura para establecer los riesgos operacionales asociados al proceso, de allí se utiliza un cuestionario a un grupo de expertos del transporte de producto terminado del sector para consolidar la identificación.

\section{B. Evaluación de los riesgos}

La evaluación de riesgos ayuda a priorizar los riesgos y a su vez, definir acciones para mitigar la vulnerabilidad de la cadena [5].

Las herramientas para la medición del riesgo pueden ser cualitativas, cuantitativas o semicuantitativas [6]; las primeras se desarrollan cuando no se dispone de suficiente información y experiencia en el pasado, los segundos son preferibles cuando los riesgos son relativamente bajos en severidad y ocurrencia y los terceros, donde se emplean herramientas analíticas, se reconocen como la mejor compensación entre las percepciones subjetivas y las técnicas rigurosas [7].

La propuesta para la evaluación de los riesgos en el proceso ya descrito, se fundamenta en la priorización mediante la construcción de la matriz probabilidad impacto y la aplicación de la metodología FQFD (despliegue de la función de calidad difusa). Es necesario resaltar que la priorización obtenida con la matriz tiene en consideración la probabilidad de ocurrencia y la severidad de materialización de cada riesgo, mientras que el FQFD prioriza en relación a los objetivos estratégicos del proceso.

\section{Evaluación de los riesgos}

La mitigación de los riesgos implica desarrollar estrategias para disminuir la probabilidad y el impacto de los mismos, las cuales deben orientar la gestión bajo condiciones normales, anormales o en grandes interrupciones haciendo de los procesos más resistentes [8]. Es así como la definición de las acciones y medidas a implementar, debe tener en cuenta los riesgos prioritarios considerándolos a partir de su alto impacto en la organización [9].

En este sentido se propone el ciclo PHVA (Planear, verificar $y$ actuar) como herramienta para proponer actividades que hagan frente a los riesgos prioritarios. La fase planear contempla la definición de la problemática, la descripción de la situación actual y el análisis de los hechos para concluir con la propuesta de las actividades.

\section{DESARROLLO DE LA METODOLOGÍA Y RESULTADOS}

Esta metodología parte de elementos encontrados en la revisión de la literatura para la gestión del riesgo y a continuación se detallan cada una de las fases. Es importante destacar que estas fases son secuenciales.

\section{A. Identificación de los riesgos}

Con la revisión de la literatura se obtuvo la lista de los riesgos operacionales presentes en el proceso de transporte terrestre de producto terminado, tal como se detalla en la tabla I. Estos riesgos identificados son corroborados a través de un cuestionario aplicado a un grupo de expertos en el proceso desde diferentes roles. 
TABLA I RIESGOS IDENTIFICADOS PARA EL PROCESO

\begin{tabular}{ll}
\hline \hline & \\
\hline R1 & Los accidentes de tránsito \\
R2 & La comunicación no efectiva \\
R3 & La contaminación del producto \\
R4 & El daño de mercancías \\
R5 & El deslizamiento \\
R6 & Las fallas mecánicas del vehículo \\
R7 & La falta de experiencia del conductor \\
R8 & La falta de planeación del transporte \\
R9 & La fatiga del conductor \\
R10 & El incendio o la explosión \\
R11 & El incumplimiento a regulaciones de tránsito \\
R12 & Incumplimiento en los tiempos de entrega \\
R13 & La negligencia del conductor \\
R14 & Los paros, huelgas y manifestaciones \\
R15 & El robo de mercancías \\
R16 & El terrorismo \\
R17 & Las vías en mal estado \\
R18 & El volcamiento \\
\hline \hline
\end{tabular}

\section{B. Evaluación de los riesgos}

En el mismo cuestionario donde se corroboró la identificación de los riesgos que se habían identificado en la literatura, los expertos en el proceso definieron también para cada uno, la probabilidad de ocurrencia y la severidad al materializarse, obteniendo así la priorización, al consolidar la información aplicando el promedio en la probabilidad, (1), y el promedio a la severidad, (2).

$$
\begin{gathered}
\overline{X p}=\frac{\sum_{i=1}^{n} x_{i} p_{i}}{n} \\
\overline{X S}=\frac{\sum_{i=1}^{n} x_{i} s_{i}}{n}
\end{gathered}
$$

Dónde:

$$
\begin{aligned}
& \overline{X p}=\text { Promedio probabilidad } \\
& \overline{X s}=\text { Promedio severidad } \\
& X_{i}=\text { Indicencia del riesgo. Si }=1 \text { No }=0 \\
& p_{i}=\text { Probabilidad de persona encuestada } \\
& s_{i}=\text { Severidad de persona encuestada } \\
& n=\text { Total personas encuestadas }
\end{aligned}
$$

Con esta información, se ilustran los resultados en la matriz probabilidad e impacto, que se muestra en la Fig. 2, donde por colores se indica el nivel crítico de cada riesgo; siendo la zona roja y naranja los riesgos críticos y a tener en cuenta para aplicar la metodología FQFD, la amarilla son los de nivel medio, finalmente la verde indica los no relevantes.

De los riesgos identificados a criterio de los expertos, sólo tres tienen un nivel medio (R7, R10, R12) y no hay asignados a los no relevantes.

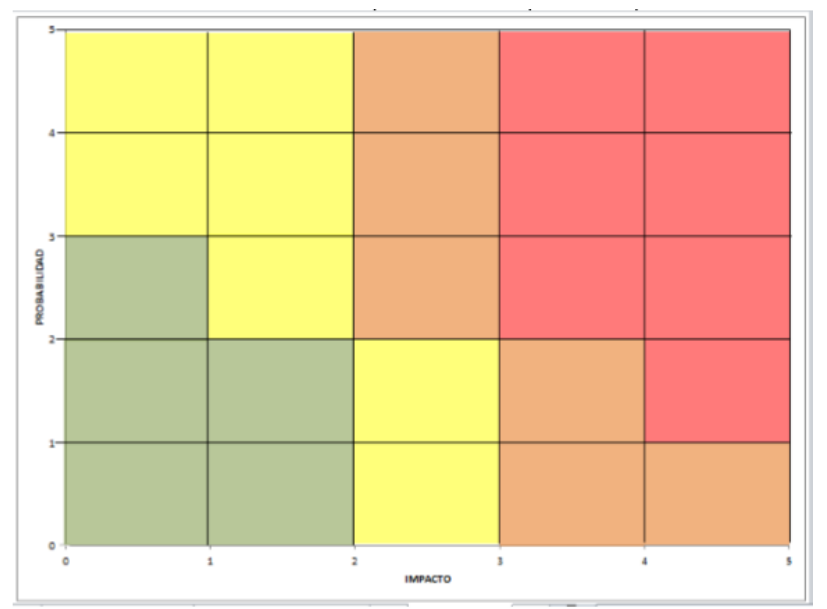

Fig. 2. Matriz probabilidad e impacto adaptada de Menéndez [10]

La priorización para riesgos operacionales a través de la metodología FQFD (Despliegue de la función de calidad difusa) propuesta por Osorio [11] consta de siete etapas, las cuales se notan en la Fig. 3.

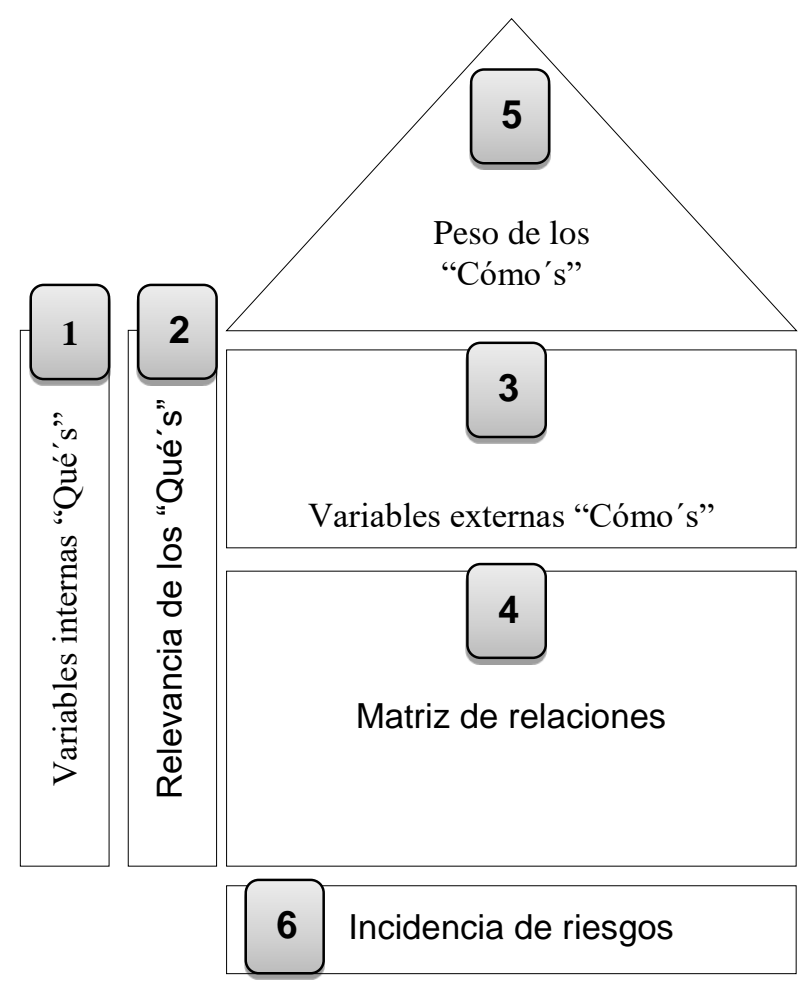

Fig. 3. Etapas del FQFD

- Paso 1: Variables internas "Que's” 
En este paso se definen los deseos del proceso desde diferentes roles involucrados. Se realiza una entrevista a cada miembro del grupo decisor y al consolidar la información se definen las prioridades del transporte de producto terminado en el sector azucarero, que se presentan en la tabla II.

TABLA II.

VARIABLES INTERNAS "QUÉ'S".

\begin{tabular}{l}
\hline \hline \multicolumn{1}{c}{ QUÉ'S } \\
\hline \hline Entrega eficiente (Costo y tiempo) \\
Conservar el producto terminado en buen estado \\
Que no se contamine el producto terminado \\
Condiciones mecánicas adecuadas del vehículo \\
Condiciones higiénicas adecuadas del vehículo \\
\hline \hline
\end{tabular}

\section{- Paso 2: Relevancia de los "Qué's"}

Para este paso, inicialmente se define la escala lingüística para que el grupo decisor realice la calificación y sus equivalentes números difusos triangulares, así como se muestra en la tabla III.

TABLA III.

ESCALA LINGÜÍSTICA Y NÚMERO DIFUSO.

\begin{tabular}{ccc}
\hline \hline & ESCALA & NÚMERO DIFUSO \\
MB & Muy Bajo & $(0,1,2)$ \\
B & Bajo & $(2,3,4)$ \\
M & Medio & $(4,5,6)$ \\
A & Alto & $(6,7,8)$ \\
MA & Muy Alto & $(8,9,10)$ \\
\hline \hline
\end{tabular}

El peso de cada variable interna es dado por todos los integrantes del grupo decisor, que al aplicar la ponderación que se muestra en (3) se obtiene el peso general de cada "Qué".

Peso $_{\mathrm{Q} u e ́}=\left\{w_{i}\right.$, dondei $\left.=1, \ldots, q\right\}$,

$w_{i}=\frac{1}{n} \otimes\left(w_{i 1} \oplus w_{i 2} \oplus \ldots \oplus w_{i n}\right) \quad$ (3)

Dónde:

Qués $=\mathrm{q}$

$n=$ número de miembros en el equipo

$w_{i}=$ número triángular $\left(w_{i a}, w_{i b}, w_{i c}\right)$

Así, se llega a la relevancia de las variables internas "Qué's" donde se obtiene un número triangular difuso asociado, esto se nota en la tabla IV.
TABLA IV.

RELEVANCIA DE LOS “QUÉ'S”

\begin{tabular}{lc}
\hline \multicolumn{1}{c}{ QUÉ'S } & PESO \\
\hline \hline Entrega eficiente (Costo y tiempo) & $(6,7,8)$ \\
Conservar el producto terminado en buen estado & $(7,8,9)$ \\
Que no se contamine el producto terminado & $(7,8,9)$ \\
Condiciones mecánicas adecuadas del vehículo & $(4,5,6)$ \\
Condiciones higiénicas adecuadas del vehículo & $(5,6,7)$ \\
\hline \hline
\end{tabular}

- Paso 3: Variables externas "Cómo's"

Los "Cómo's" hacen referencia a los objetivos estratégicos relacionados con el proceso. Para definirlos, se tiene en cuenta que el grupo multidisciplinario se conformó a partir de diferentes roles del proceso de producto terminado del azúcar.

Se revisaron entonces las misiones de las organizaciones y a partir de esta información, se identificaron aspectos claves relacionados con los objetivos estratégicos del proceso, posteriormente se consultó con los expertos, los cuales corroboraron dichas relaciones. Finalmente se consolidó la información en la tabla $\mathrm{V}$, donde están los objetivos a tener en cuenta para el desarrollo del FQFD.

TABLA V.

VARIABLES EXTERNAS “CÓMO'S"

\section{CÓMO’S}

\begin{tabular}{ll}
\hline \hline Objetivo 1 & Preservar la calidad del producto \\
objetivo 2 & Competitividad de la organización \\
Objetivo 3 & Cumplir requerimientos de clientes \\
Objetivo 4 & Generar valor a los accionistas \\
Objetivo 5 & Inocuidad del producto \\
\hline \hline
\end{tabular}

- $\quad$ Paso 4: Matriz de relaciones

La relación se establece entre los “Qué's" siendo las variables internas y los "Cómo's" haciendo referencia a las variables externas del proceso. En este caso cada miembro, calificó la relación entre estas variables y teniendo en cuenta (4) se obtuvo la matriz de relación.

$$
\begin{aligned}
& \text { Relación }=\left\{r_{i j} \quad \text { dónde } e_{j=1, \ldots, c}^{i=1, ., q}\right\}, \\
& \mathrm{r}_{\mathrm{ij}}=\frac{1}{\mathrm{n}} \otimes\left(\mathrm{r}_{\mathrm{ij} 1} \oplus \mathrm{r}_{\mathrm{ij} 2} \oplus \ldots \oplus \mathrm{r}_{\mathrm{ijn}}\right) \\
& \text { Dónde: } \\
& \text { Cómo's }=\mathrm{c} \\
& \text { Qué's }=\mathrm{q}
\end{aligned}
$$


$\mathrm{n}=$ Número de integrante sdel equipo

$r_{i}=$ número triángular $\left(r_{i j a}, r_{i j b}, r_{i j c}\right)$

consolidado entre cada $=i-Q u e ́$ y $j-$ Cómo

De este modo, la matriz de relación para este proceso se evidencia en la tabla VI.

TABLA VI.

MATRIZ DE RELACIONES

\begin{tabular}{llllll}
\hline \hline \multicolumn{5}{c}{ RELACIÓN QUÉ/CÓMO } \\
\hline \hline \multirow{5}{*}{ Objetivo 1 } & Q1 & Q2 & Q3 & Q4 & Q5 \\
& 6,5 & 7 & 6 & 4,5 & 6 \\
& 8,5 & 8 & 7 & 5,5 & 7 \\
& 6,5 & 9 & 8 & 6,5 & 8 \\
Objetivo 2 & 7,5 & 7 & 6,5 & 5 & 5,5 \\
& 8,5 & 8 & 7,5 & 6 & 6,5 \\
& 7,5 & 8 & 7,5 & 5 & 5,5 \\
Objetivo 3 & 8,5 & 9 & 8,5 & 6 & 6,5 \\
& 9,5 & 10 & 9,5 & 7 & 7,5 \\
& 7,5 & 6,5 & 5,5 & 4,5 & 4 \\
Objetivo 4 & 8,5 & 7,5 & 6,5 & 5,5 & 5 \\
& 9,5 & 8,5 & 7,5 & 6,5 & 6 \\
& 6 & 7,5 & 7,5 & 2,5 & 7 \\
Objetivo 5 & 7 & 8,5 & 8,5 & 3,5 & 8 \\
& 8 & 9,5 & 9,5 & 4,5 & 9 \\
\hline \hline
\end{tabular}

- Paso 5: Peso de los “Cómo's”

Al recopilar la información de la relevancia de los "Qué's" y el ponderado de la relación "Qué"-“Cómo", se obtiene el peso relativo de los "Cómo's", esto se hace a manera de (5).

Peso $_{\text {como }}=\left\{\mathrm{W}_{\mathrm{J}}\right.$, dondej $\left.=1, \ldots, \mathrm{c}\right\}$,

TABLA VIII

INCIDENCIA DELOS RIESGOS

$\mathrm{W}_{\mathrm{j}}=\frac{1}{\mathrm{q}} \otimes\left[\left(\mathrm{r}_{\mathrm{j} 1} \otimes \mathrm{w}_{1}\right) \oplus \ldots \oplus\left(\mathrm{r}_{\mathrm{jq}} \otimes \mathrm{w}_{\mathrm{q}}\right)\right]$

Dónde:

Cómo's = c

Qué's $=\mathrm{q}$

$w_{j}=$ número triángular $\left(w_{j a}, w_{j b}, w_{j c}\right)$

TABLA VII.

PESO DE LOS "CÓMO'S"

\begin{tabular}{lccc}
\hline \hline \multicolumn{4}{c}{ PESO DE LOS CÓMO’S } \\
\hline \hline Objetivo 1 & $\mathbf{3 5 , 6}$ & $\mathbf{4 8}$ & $\mathbf{6 3}$ \\
Objetivo 2 & $\mathbf{3 1 , 6}$ & $\mathbf{4 4}$ & $\mathbf{5 8}$ \\
Objetivo 3 & $\mathbf{4 0 , 2}$ & $\mathbf{5 4}$ & $\mathbf{6 9}$ \\
Objetivo 4 & $\mathbf{3 3 , 4}$ & $\mathbf{4 6}$ & $\mathbf{6 0}$ \\
Objetivo 5 & $\mathbf{3 7 , 2}$ & $\mathbf{5 0}$ & $\mathbf{6 5}$ \\
\hline \hline
\end{tabular}

- Paso 6: Incidencia de los riesgos

Aquí cada integrante del grupo determinó la incidencia de los riesgos identificados en el proceso con los objetivos definidos, esto se hizo mediante (6).

$I R=\left\{\begin{array}{c}I_{h J}, \text { dondeh }^{\prime}=1, \ldots, p, \\ j=1, \ldots, c\end{array}\right\}$,

$\mathrm{IR}_{\mathrm{hj}}=\frac{1}{\mathrm{n}} \otimes\left(\mathrm{ir}_{\mathrm{hj} 1} \oplus \ldots \oplus \mathrm{ir}_{\mathrm{hjn}}\right)$

Dónde:

$\mathrm{p}=$ Número de riesgos identificados

$\mathrm{n}=$ Número de integrante sdel equipo $i r_{h j n}=$ Evalución del riesgo h según objetivo $j$

De esta manera se obtuvo la incidencia de cada riesgo en los objetivos estratégicos del proceso en número difuso, esto se presenta en la tabla VIII.

Cabe destacar que en este punto no acaba la priorización, la metodología FQFD se complementa con la desfusificación de los números triangulares y la definición de los rangos de prioridad.

\begin{tabular}{llll}
\hline \hline \multicolumn{3}{c}{ INCIDENCIA DE RIESGOS } \\
\hline \hline R1 & $\mathbf{1 9 6 , 1}$ & $\mathbf{3 1 4 , 5}$ & $\mathbf{4 7 3 , 5}$ \\
R2 & $\mathbf{2 1 4 , 6}$ & $\mathbf{3 3 9 , 6}$ & $\mathbf{5 0 6}$ \\
R3 & $\mathbf{2 5 0 , 7}$ & $\mathbf{3 8 8 , 4}$ & $\mathbf{5 6 9 , 6}$ \\
R4 & $\mathbf{2 4 0 , 1}$ & $\mathbf{3 7 4 , 1}$ & $\mathbf{5 5 0 , 9}$ \\
R6 & $\mathbf{1 6 0 , 1}$ & $\mathbf{2 6 5 , 7}$ & $\mathbf{4 0 9 , 8}$ \\
R8 & $\mathbf{1 9 1 , 2}$ & $\mathbf{3 0 8 , 2}$ & $\mathbf{4 6 5 , 5}$ \\
R9 & $\mathbf{1 3 4 , 6}$ & $\mathbf{2 3 1 , 3}$ & $\mathbf{3 6 5}$ \\
R11 & $\mathbf{1 3 0 , 6}$ & $\mathbf{2 2 5 , 9}$ & $\mathbf{3 5 8 , 1}$ \\
R13 & $\mathbf{1 5 3 , 7}$ & $\mathbf{2 5 6 , 9}$ & $\mathbf{3 9 8 , 2}$ \\
R14 & $\mathbf{1 5 8 , 5}$ & $\mathbf{2 6 3 , 3}$ & $\mathbf{4 0 6 , 3}$ \\
R15 & $\mathbf{1 5 3 , 5}$ & $\mathbf{2 5 6 , 8}$ & $\mathbf{3 9 8 , 1}$ \\
R16 & $\mathbf{1 7 0 , 3}$ & $\mathbf{2 7 9 , 7}$ & $\mathbf{4 2 8 , 3}$ \\
R17 & $\mathbf{1 9 7}$ & $\mathbf{3 1 5 , 6}$ & $\mathbf{4 7 4 , 7}$
\end{tabular}


R18

182,9

296,4

449,6

Haciendo uso de los números triangulares, se establecieron los límites, como se muestran en la tabla IX, que permitieron clasificar y visualizar los riesgos por color, dónde el rojo indica acción inmediata.

TABLA IX

RANGO DE CRITICIDAD

\begin{tabular}{|c|c|c|c|c|}
\hline \multirow{2}{*}{$\begin{array}{l}\text { NIVEL } \\
\text { MA }\end{array}$} & \multicolumn{3}{|c|}{ LÍMITES TRIANGULARES } & \multirow{2}{*}{$\begin{array}{r}\text { LÍMITES } \\
\mathbf{4 4 6 , 4 3}\end{array}$} \\
\hline & 284,8 & 435,1 & 630,8 & \\
\hline A & 213,6 & 338,4 & 504,6 & 348,75 \\
\hline M & 142,4 & 241,7 & 378,5 & 251,07 \\
\hline B & 71,2 & 145 & 252,3 & 153,39 \\
\hline MB & $\mathbf{0}$ & 48,3 & 126,2 & 55,71 \\
\hline
\end{tabular}

Con estos rangos y junto a la desfuzificación de la incidencia de los riesgos, donde los números triangulares se convierten en uno solo y se genera el índice de prioridad, se presentan en la tabla X la clasificación de riesgos.

Es así como a través de una serie de entrevistas con cada miembro del grupo decisor y al consolidar la información se definen los riesgos operacionales prioritarios del transporte de producto terminado en el sector azucarero.

TABLA X.

RIESGOS PRIORITARIOS

\begin{tabular}{lll}
\hline \multicolumn{2}{c}{ PRIORIZACIÓN DE RIESGOS } \\
\hline \hline R3 & La contaminación del producto & $\mathbf{3 9 9}$ \\
R4 & El daño de mercancías & $\mathbf{3 8 5}$ \\
R2 & La comunicación no efectiva & $\mathbf{3 5 0}$ \\
R17 & Las vías en mal estado & $\mathbf{3 2 6}$ \\
R1 & Los accidentes de tránsito & $\mathbf{3 2 5}$ \\
R8 & La falta de planeación del transporte & $\mathbf{3 1 8}$ \\
R18 & El volcamiento & $\mathbf{3 0 6}$ \\
R16 & El terrorismo & $\mathbf{2 9 0}$ \\
R6 & Las fallas mecánicas del vehículo & $\mathbf{2 7 5}$ \\
R14 & Los paros, huelgas y manifestaciones & $\mathbf{2 7 3}$ \\
R13 & La negligencia del conductor & $\mathbf{2 6 6}$ \\
R15 & El robo de mercancías & $\mathbf{2 6 6}$ \\
R9 & La fatiga del conductor & $\mathbf{2 4 1}$ \\
R11 & El incumplimiento a regulaciones de tránsito \\
\hline \hline
\end{tabular}

\section{Acciones orientadas a la mitigación}

En esta etapa, es importante tener en cuenta el entorno de cada organización en la que se presenta el proceso. Con los riesgos ya priorizados es necesario identificar cuáles son las causas que pueden generar la materialización.

Una herramienta útil es el diagrama de Ishikawa, que permite contextualizar los riesgos presentes en el proceso y a partir de allí definir el plan de acción para cumplir con la gestión del riesgo.

\section{CONCLUSIONES}

Un proceso como el transporte de producto terminado en el sector azucarero, involucra personas de diferentes roles para su cumplimiento, cada una tiene una percepción, unos deseos y unos objetivos para este proceso desde su rol y no necesariamente son los mismos. El desarrollo de este trabajo permitió considerar e integrar la experiencia de diferentes roles para el transporte de producto terminado para deliberar acerca de cuáles son los riesgos que pueden afectar el proceso.

De los riesgos identificados a partir de la revisión bibliográfica, el $77,7 \%$ son identificados como riesgos de criticidad alta, bajo la opinión de las personas que tienen experiencia en el proceso de transporte de producto terminado,

Con la herramienta FQFD se estableció la prioridad de los riesgos, no sólo teniendo en cuenta la experiencia de las personas involucradas en el proceso, sino que también se consideró la relación de estos riesgos con los objetivos de las organizaciones.

Los riesgos prioritarios a considerar para el proceso de transporte de producto terminado son, la contaminación del producto, el daño y la comunicación no efectiva, para los cuales se determinó cuáles eran las posibles causas con base en revisión literaria y opinión de personas involucradas en el proceso.

De los riesgos priorizados, el 35,7\% tienen un alcance de control externo a las organizaciones del sector, pero es importante mapearlos con el fin de establecer un plan de contingencia al momento que se puedan materializar.

La metodología propuesta garantiza que al ser aplicada en una organización va a contribuir a una gestión del riesgo efectiva.

\section{REFERENCIAS}

[1] F. Wiengarten, P. Humphreys, C. Gimenez, and R. McIvor, "Risk, risk management practices, and the success of supply chain integration," Int. J. Prod. Econ., vol. 171, pp. 361-370, 2016.

[2] PROCAÑA, "Presentación del Sector agroindustrial de la Caña de Azúcar," Procaña, 2018.

[3] J. (QSL consultores) Castellanos, "Gestión del riesgo operacional," 2007, pp. 32, 33.

[4] M. Delfiner and C. Pailhé, "Qualitative techniques for managing operational risk," no. 75599, 2009.

[5] J. C. Osorio, D. F. Manotas, and L. Rivera, "Priorización de Riesgos Operacionales para un Proveedor de Tercera Parte Logística - 3PL," Inf. tecnológica, vol. 28, no. 4, pp. 135-144, 2017.

[6] P. Singhal, G. Agarwal, and M. L. Mittal, "Supply chain risk management: Review, classification and future research directions," Int. J. Bus. Sci. Appl. Manag., vol. 6, no. 3, pp. 15-42, 2011.

[7] H. Zhu, C. Li, C. Mo, F. Jiang, and M. Zhang, TEM characterization of defects in GaN/InGaN multi-quantum wells grown on silicon by MOCVD, vol. 29, no. 3. 2008.

[8] M. Christopher, "Managing risk in the global supply chain," Logist. Supply Chain Manag., pp. 231-58, 2014.

[9] J. C. Osorio Gómez, "Gestión del riesgo operacional en cadenas de suministro considerando 3PL." 2017.

[10] FAO, "Pérdidas y desperdicios de alimentos en América Latina y el 


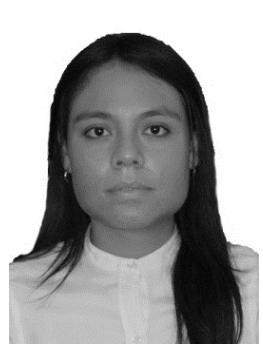

2019.

Laboró como Asistente Administrativa de 2012 a 2014 y realizó sus prácticas profesiones, como estudiante de ingeniería en el Ingenio Manuelita para el período de junio a diciembre de 2018.

\section{ORCID:}

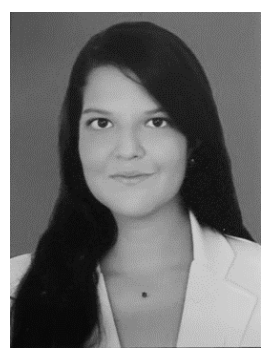

M. Colorado, Nace en la ciudad de Tuluá Valle el 05 de septiembre de 1996. En el 2014 se titula como técnico en operaciones comerciales y financieras del SENA, posteriormente en el 2019 se titula como ingeniera industrial de la universidad del Valle.

Posee experiencia como auxiliar administrativa en Tecnotaxia, facilitadora en la implementación de TPM y LEAN en Nestlé y actualmente se desempeña como analista de soporte logístico en el centro de contacto de Coomeva.

Orientada a la mejora continua, planeación, trabajo en equipo, análisis de la información y toma de decisiones

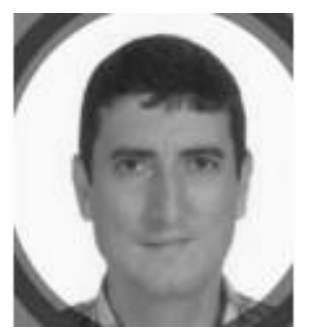

J.C. Osorio-Gómez, nacido el 20 de septiembre de 1975 en Buga, Valle del Cauca, Colombia. Ingeniero Industrial, Especialista en Logística, Magister en Ingeniería Industrial, Doctor en Ingeniería Industrial de la Universidad del Valle, Santiago de Cali, Valle del Cauca, Colombia. Actualmente Profesor titular de la Escuela de Ingeniería Industrial de la Universidad del Valle.

ORCID: http://orcid.org/0000-0001-5625-5609 